\title{
Semana de conscientização sobre o cérebro em Botucatu: Interação universidade e ensino fundamental
}

\author{
Juliana Irani Fratucci De Gobbi ${ }^{1}$, Ana Carolina Inhasz Kiss ${ }^{2}$, Silvia Mitiko Nishida ${ }^{3}$
}

\begin{abstract}
Resumo: A população carece de boas fontes de informações sobre temas relacionados às neurociências. Eventos de extensão podem contribuir para divulgar e estimular o gosto pelo campo de estudo desde o ensino fundamental. Neste artigo relatamos nossas experiências durante três anos consecutivos de eventos nas semanas de conscientização sobre o cérebro em Botucatu. As principais ações foram realizadas em um educandário e em um projeto ligado à ação da cidadania do município. As ações incluíram: (i) atividades lúdicas seguidas de gincana de perguntas e respostas para fixação do conteúdo trabalhado; (ii) distribuição de palavras cruzadas, caças palavras, labirintos e folhas para colorir sobre os temas abordados para as crianças e para usuários de um restaurante popular localizado ao lado do hospital das clínicas do campus; (iii) palestra noturna sobre o tema da semana para pais e amigos das crianças do educandário. As ações tiveram grande aceitação por parte das crianças, alunos (7-14 anos), professores e adultos. Estimamos que houve, nos três anos, envolvimento de cerca de 590 crianças e 600 adultos, atingindo o objetivo de promover a popularização da neurociência em âmbito escolar e público, aproximando comunidade e universidade.
\end{abstract}

Palavras-chave: Popularização da Ciência; Cérebro; Lúdico; Neurociências

\section{Botucatu Brain Awareness Week: University and elementary school interaction}

Abstract: The population lacks good sources of information on neuroscience topics. Outreach events can contribute to this dissemination and stimulate a taste for neurosciences since elementary school. In this article, we report our experiences over three consecutive years of events in Brain Awareness Week in Botucatu. The main actions of the events were carried out in an school and a municipality project related to citizenship. The actions included: (i) play activities followed by a question and answer session to fix the content worked; (ii) distribution of crosswords, word hunts, labyrinths and coloring sheets on topics addressed to children and users of a popular restaurant, located next to the clinic hospital of the campus, (iii) evening talk for parents and friends (200 adults) of the children studying in the school, about the topic of the week. The actions were widely accepted by the children, students (7-14 years old), teachers, and adults participating. We estimate in the three years about 590 children and 600 adults, achieving the goal of promoting the popularization of neuroscience in school and public, approaching the university community.

Keywords: Popularization of Science; Brain; Playful; Neuroscience
Originais recebidos em

23 de janeiro de 2019

Aceito para publicação em

14 de março de 2020

1

Universidade Estadual Paulista UNESP. Doutora em Fisiologia, docente do Departamento de Fisiologia do Instituto de Biociências de Botucatu.

juliana.gobbi@unesp.br (autora para correspondência)

2

Universidade Estadual Paulista UNESP. Doutora em Fisiologia, docente do Departamento de Fisiologia do Instituto de Biociências de Botucatu. ana.inhasz@unesp.br 3 Universidade Estadual Paulista UNESP. Doutora em Fisiologia, docente do Departamento de Fisiologia do Instituto de Biociências de Botucatu. silvia.nishida@unesp.br 


\section{Introdução}

Neurociências é um campo maravilhoso e intrigante, sendo o ponto de intersecção de várias áreas, tanto daquelas diretamente relacionadas às clássicas anatomia e fisiologia, quanto das que tratam do comportamento humano nas mais variadas nuances. Entender o sistema nervoso sempre gera fascínio, curiosidade e inquietação por ser algo complexo, visto que o próprio cérebro está se investigando. Muitas vezes interpretações equivocadas de fatos científicos sobre a mente ou o cérebro culminam nos "neuromitos" (Pasquinelli, 2012). Um dos papéis da universidade é o compromisso com a veiculação de informações corretas, colaborando para evitar que conhecimentos falsos façam parte do cotidiano da população, prevenindo que sejam perpetuadas informações distorcidas e equivocadas. Um mito bem conhecido é que usamos apenas 10\% do nosso cérebro. A pesquisadora Herculano-Houzel (2002), em uma enquete feita com a população carioca, evidenciou que esse "neuromito" ainda era aceito como verdade mesmo no século XXI e ao final da chamada década do cérebro. Esta correspondeu à década de 1990, quando houve um avanço intenso sobre as pesquisas em neurociências, bem como um aumento de disseminação de informações pela mídia para a sociedade (Racine et al., 2010). Isto nos chama atenção para a necessidade de uma divulgação bem estruturada de conhecimentos para a população, de forma mais clara e contínua, para que sejam sanadas as distorções existentes e não sejam criadas novas. Entre universitários de uma mesma instituição, os estudantes de psicologia são mais céticos quanto a palavras relacionadas ao cérebro, acreditando menos em "neuromitos" que outros estudantes (Lindell \& Kidd, 2013).

As neurociências encontram mistificações mesmo no ensino superior; a complexidade do estudo acadêmico sobre o tema popularizou o termo "neurofobia" entre estudantes dos cursos de Medicina e afins. Este fato comprova a ansiedade dos estudantes com a área do conhecimento, em especial nas disciplinas sobre o cérebro relacionadas à Neuroanatomia e Neurofisiologia (Abushouk \& Duc, 2016; Santos-Lobato et al., 2018). Tal ansiedade gera tanto desconforto nos estudantes, que atinge a qualidade e o número de neurologistas formados (Abushouk \& Duc, 2016; Buonanotte et al., 2016). Desta forma, a disseminação mais precoce sobre os conceitos neurocientíficos, desde o ensino fundamental, de forma lúdica e interativa, poderá contribuir para mitigar a ansiedade sobre o tema, não apenas para possíveis estudantes de Medicina, mas também para futuros professores do ensino básico, formados nos cursos de licenciatura. Acreditamos que, quanto mais cedo for despertado gosto e interesse por neurociências nos escolares, menores as chances de "neuromitos" se perpetuarem.

Segundo Candotti (2002), a divulgação científica é uma das responsabilidades sociais dos cientistas. Eventos pautados na divulgação de conhecimentos são bons momentos para acessar conceitos que a população em geral possui sobre um assunto e inserir correções e ideias novas ao respeito (Fragel-Madeira \& Aranha, 2012). Despertar o gosto pelo conhecimento científico já no ensino fundamental é crucial e estratégico para incentiválos na continuidade dos estudos, especialmente em regiões socialmente mais vulneráveis. Ressaltamos uma observação de um eminente neurocientista brasileiro, Roberto Lent (2009), que faz uma analogia e uma reflexão sobre o papel da divulgação científica para crianças: "O Brasil é bom de futebol porque $90 \%$ dos meninos praticam o esporte. Que ótimo seria se essas crianças também estivessem envolvidas com ciência!". Incentivar o estudo e divulgar conhecimentos é uma forma de despertar as pessoas para o mundo que as cercam. Disseminar conhecimentos científicos, compartilhar informações para a comunidade é um dos papéis da extensão universitária, o que ajuda no despertar de pessoas.

No mês de março, em geral na segunda semana, é comemorada mundialmente a Semana de Conscientização sobre o Cérebro. Essa campanha foi idealizada pela Dana Foundation, uma organização filantrópica americana, em 1996. O objetivo principal é aumentar a consciência pública sobre os progressos e benefícios das pesquisas 
do cérebro. A organização cadastra on-line parceiros em todo o mundo que ajudam em atividades de divulgação científica voltadas para pessoas de todas as idades. Na campanha de 2018 participaram das atividades 42 países. O Departamento de Fisiologia do Instituto de Biociências de Botucatu vem participando há três anos das atividades. Existem várias possibilidades para a exposição de um tema. Para as atividades dessa semana escolhemos a divulgação por meio do lúdico e do interativo, com atividades do tipo "mãos à obra". Estas são incentivadas pela Dana Foundation, e já foram documentadas como muito proveitosas pelas crianças (Zardetto-Smith et al., 2002; Filipin et al., 2014).

O objetivo deste artigo é relatar a nossa experiência na divulgação sobre neurociências, representando no município de Botucatu a multiplicação de ações do Dana Foundation, tendo como alvo o público de ensino fundamental e a população em geral.

\section{Materiais e Métodos}

\section{População}

Nosso público alvo foi crianças do ensino fundamental do Educandário Professor Eurípedes Barsanulfo do Núcleo Assistencial Joanna de Ângelis, e do projeto Preservando o Futuro, uma organização não governamental ligada à Ação da Cidadania do município de Botucatu, duas comunidades de maior vulnerabilidade, nas periferias da cidade. Também foram abordados pacientes, familiares, servidores e estudantes do campus da UNESP de Botucatu que fazem suas refeições no restaurante Bom Prato, e os pais e amigos dos alunos do educandário em palestras noturnas.

\section{Procedimentos}

Partindo da premissa de que estratégias eficazes na divulgação de conhecimentos seriam por meio de propostas lúdicas e interativas usando o contexto do cotidiano do aprendiz, as atividades propostas foram via jogos, demonstrações, analogias com situações familiares do dia-a-dia, o que dá significado à informação, e modelos anatômicos. $\mathrm{O}$ uso de propostas lúdicas é incentivado por vários educadores a partir das ideias do pensador russo Vygotsky, que incentiva uso do lúdico como forma de ajudar a construir significados em crianças (Rolim et al., 2008).

Participaram das atividades alunos voluntários de graduação dos cursos de Ciências Biológicas, Ciências Biomédicas, Física Médica e Medicina, colaborando como produtores de materiais ou moderadores das estações de conhecimento durante o evento, além de alguns alunos de pós-graduação. As atividades foram cadastradas como evento de extensão na Pró-Reitoria de Extensão Universitária da UNESP e teve apoio do Instituto de Biociências de Botucatu.

A organização agendou dias e horários com as instituições participantes. As atividades eram pautadas em uma breve apresentação sobre as comemorações mundiais da Semana de Conscientização sobre o Cérebro, seguida de uma interação de quinze minutos de perguntas e respostas sobre o tema a ser abordado, com a intenção de despertá-los para as atividades. Em seguida, as crianças foram divididas em cinco grupos que se rodiziaram em estações com diferentes atividades sobre o tema da semana. As crianças permaneceram por quinze minutos em cada atividade. Depois os alunos foram convidados a se dividirem em três grupos e participaram de uma gincana de perguntas e respostas sobre o rodízio das estações.

Para cada estação de conhecimento foram montadas atividades demonstrativas com modelos anatômicos, lúdicas e interativas. Os cartazes utilizados foram confeccionados em papéis coloridos e com recortes em relevo com espuma vinílica acetinada (EVA). Foram confeccionados materiais de apoio e divulgação sobre o 
tema, como caça-palavras, palavras cruzadas, labirintos e materiais para colorir, que foram entregues aos professores para serem distribuídos após as atividades em sala de aula. Para a população adulta abordada no Bom Prato e aos participantes das palestras também foram distribuídos folders informativos sobre doenças neurodegenerativas, acidente vascular cerebral e as consequências do uso de algumas drogas ilícitas para o sistema nervoso.

\section{Resultados e Discussão}

Os resultados serão apresentados descrevendo-se as semanas temáticas desenvolvidas.

\section{Semana 1: Como sentimos o mundo?}

Para mobilizar a atenção dos alunos sobre os sentidos do corpo, utilizamos o Zé Cabeção, modelo interativo constituído de cabeça e cérebro produzidos com resina. O Zé Cabeção foi idealizado pela Professora Doutora Silvia Mitiko Nishida, do Departamento de Fisiologia do Instituto de Biociências, para uso em atividades didáticas e projetos de extensão. As regiões específicas do cérebro que correspondem a áreas corticais sensoriais são acesas (por uma lâmpada interna) quando estímulos são aplicados nos respectivos órgãos sensoriais. Cada lobo cerebral está pintado de uma cor diferente para que as regiões sejam facilmente identificadas. Um voluntário foi convidado a cumprimentar o boneco falando próximo à sua orelha e o lobo temporal ficou aceso bilateralmente, assim como quando a luz de um celular iluminava o olho e o lobo occipital foi aceso, e sucessivamente para os demais sentidos. Esse era o momento para explicar que o cérebro é a parte do corpo que, junto com os órgãos sensoriais, proporciona o conhecimento do mundo. Na Figura 1, um dos monitores usando o Zé Cabeção na abertura da semana.
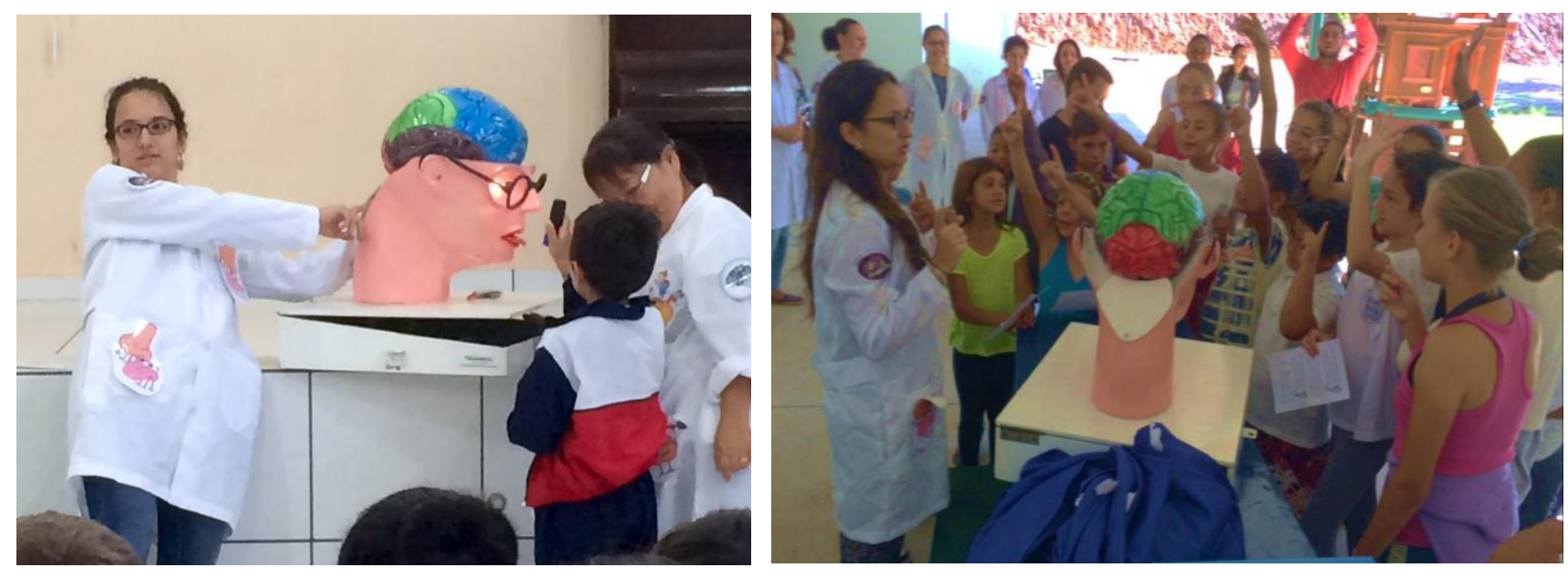

Figura 1. Utilização do boneco Zé Cabeção para mobilização da atenção dos alunos para as atividades relacionadas ao estudo dos sentidos.

Com a finalidade de favorecer a fixação das informações, usamos uma versão modificada de uma canção infantil de domínio público que foi dedilhada ao violão e cantada por uma das monitoras, incentivando as crianças a repetirem. Abaixo segue a versão da música: 
Os meus olhos, os meus olhos,

onde estão? Aqui estão!

Uso o lobo occipital, lobo occipital,

pra enxergar, vocês todos contemplar.

Minhas mãos, minhas mãos,

onde estão? Aqui estão!

Uso o lobo parietal, lobo parietal,

pra sentir, para o tato existir.

Minhas orelhas, minhas orelhas,

onde estão? Aqui estão!

Uso o lobo temporal, lobo temporal,

para escutar, sua voz apreciar.

Meu nariz, meu nariz,

onde está? Aqui está!

Uso o lobo temporal, lobo temporal

para cheirar, saber que o bolo pronto está!

Minha língua, minha língua,

onde está? Aqui está!

Uso o lobo parietal, lobo parietal

para degustar o bolo que a mãe faz.

Para cada sentido foram utilizados modelos anatômicos ou figuras didáticas. Os próprios monitores construíram um disco de Newton para demonstrar a composição da luz visível e informar que enxergamos as cores refletidas da superfície dos objetos. Para o sentido do tato foi utilizada uma "caixa surpresa" de forma que, por meio da estereognosia, as crianças pudessem nomear os objetos pela manipulação. Para mostrar que as partes do corpo não possuem a mesma sensibilidade, realizamos o teste de discriminação entre dois pontos. Este consiste em colocar simultaneamente um compasso de ponta rombuda sobre a pele com abertura diferente em diferentes partes do corpo, tendo como objetivo identificar a menor distância percebida entre dois pontos. Essa informação foi associada a como o cérebro faz a discriminação, usando uma réplica do homúnculo de Penfield produzida por um dos monitores com massa de porcelana fria. Para o sentido da audição, foram utilizados chocalhos contendo diferentes materiais que produziam sons diferentes, e as crianças adivinhavam qual era a fonte sonora. Utilizamos uma harpa de brinquedo para demonstrar as diferentes frequências dos sons; a modulação da voz (intensidade e frequência) foi utilizada para mostrar como a comunicação verbal humana pode ser afetada. Para os sentidos da gustação e olfação foram utilizadas balas de diferentes sabores (ácidas, doces, refrescantes) e texturas. Nessa ocasião apontávamos a importância dos estímulos olfatórios para compor o sabor dos alimentos. Para a olfação, foram utilizadas fontes aromáticas embebidas em algodão contendo diferentes essências, e pedíamos para relatarem os tipos de memórias evocadas. Os alunos receberam um desenho esquemático do cérebro para colorirem os lobos nas mesmas cores do Zé Cabeção, como uma forma de fixação dos conhecimentos. Nessa semana, em quatro apresentações, atingimos cerca de 240 crianças. Nosso público no educandário foi de crianças em torno dos sete anos, e no projeto da ação da cidadania, crianças entre sete e treze anos.

A gincana constava de perguntas e respostas relativas ao conteúdo apresentado sobre os órgãos do sentido e como as partes do cérebro estão associadas. A proporção de respostas corretas foi surpreendente, mostrando que o conhecimento pode ser construído efetivamente por meio da arte-educação e da interação lúdica. Foi muito satisfatório observar que as crianças haviam apreendido e entendido a divisão dos lobos 
cerebrais para cada sentido, que entenderam os componentes básicos do sistema nervoso e se sentiram recompensadas por prestarem atenção e conseguirem responder as questões, demonstrando também curiosidade e interesse sobre o que viria a seguir.

Também fizemos uma divulgação "corpo a corpo", distribuindo panfletos explicativos, desenhos para colorir e caça-palavras sobre o tema da semana para os usuários do restaurante Bom Prato do Campus de Botucatu na unidade de Rubião Junior. Enquanto os usuários aguardavam na fila, conversamos e expusemos cartazes para os interessados.

\section{Semana 2: Onde nascem as emoções?}

Para as atividades sobre as emoções fizemos uma mobilização com emojis e uma breve explanação sobre a universalidade de algumas expressões faciais, como as seis emoções básicas: alegria, medo, tristeza, raiva, surpresa e nojo. Essa divisão sobre as emoções básicas provém das pesquisas realizadas por Paul Ekman e colaboradores em diversos países (Ekman \& Friesen, 1971). Entre as estações de conhecimento tivemos: 1) explicações sobre a morfologia dos neurônios e sua propriedade excitável; 2) anatomia macroscópica do sistema nervoso; 3) neurônios, espelhos e empatia; e 4) reconhecimento de expressões faciais e neurotransmissores envolvidos nas emoções. Na Figura 2 temos uma visão geral sobre as estações. Utilizamos como modelo e inspiração para a estação 4 o livro Desenhando emoções (Sholl-Franco et al., 2015). A gincana foi realizada com jogo de perguntas e respostas como se os estudantes fossem os neurotransmissores de um neurônio que, à medida que respondessem corretamente as perguntas, poderiam se agrupar no terminal axônico. Na edição da segunda semana alcançamos 150 alunos.

Para o público adulto foram realizadas duas palestras, uma sobre "Efeitos dos agentes poluidores do meio ambiente sobre o sistema cerebral que controla as emoções", proferida pelo pesquisador Doutor Francisco Antonio Godinho, do Centro de Assistência Toxicológica (CEATOX), vinculado ao Instituto de Biociências de Botucatu, UNESP. A outra palestra foi sobre os "Benefícios da meditação mindfulness para o entendimento das próprias emoções", proferida por uma instrutora no assunto do grupo Assertiva Mindfulness de Botucatu. As palestras foram proferidas no auditório da entidade filantrópica, em dias alternados, e o público foi em torno de 200 pessoas em cada uma.
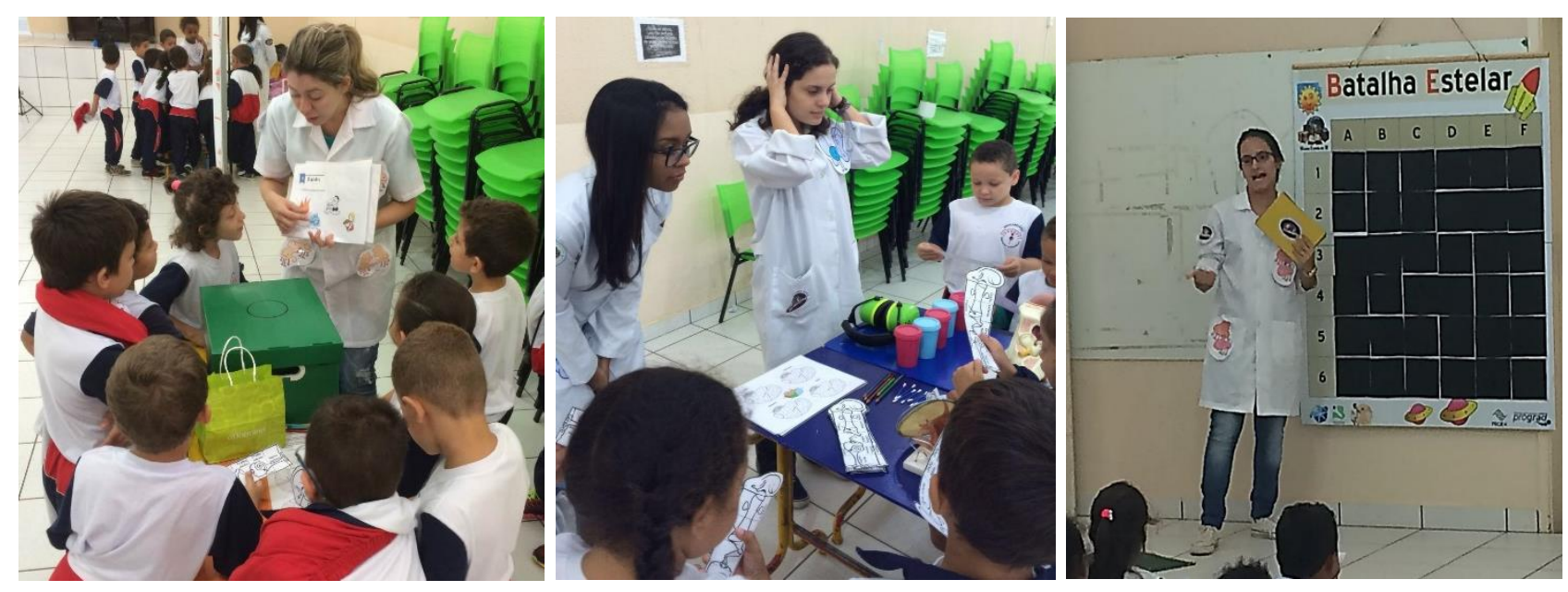

Figura 2. Detalhe das apresentações nas estações de conhecimento sobre o tato, audição e a gincana para consolidação das informações em formato de "Batalha Estelar", uma adaptação do jogo batalha naval. 


\section{Semana 3: "Você sabe da importância do sono?"}

Durante a terceira semana a mobilização para o tema foi feita por meio de algumas perguntas sobre o conhecimento dos alunos a respeito do sono e em seguida passamos para as estações do conhecimento. Tivemos como estações: 1) explicações sobre diferenças da massa cinzenta e massa branca do cérebro; 2) o que são as sinapses e os principais neurotransmissores; 3) importância do sono para a memória com jogo de cartas sobre hábitos positivos e hábitos que devem ser evitados para uma boa noite de sono; 4) tipo de ondas cerebrais e importância da glândula pineal na secreção da melatonina, o hormônio do sono, bem como um gif sobre o que é o rapid eye movement (fase REM, movimento rápido dos olhos). Utilizamos muitos cartazes com relevo e demonstrações. Atingimos em torno de 200 alunos, em 3 dias de apresentações. A Figura 3 mostra o momento em que as crianças manipulam um dos modelos anatômicos usados durante as estações.

Para o público adulto foi realizada uma palestra sobre a importância do sono e de hábitos relacionados à higiene do sono para a saúde, que contou com aproximadamente 200 pessoas. Nessa terceira versão também foram realizadas palestras para 250 alunos de um cursinho pré-vestibular vinculado à Faculdade de Medicina de Botucatu sobre a temática da importância do sono. Para eles também foram distribuídos panfletos e caçapalavras sobre a temática sono.

\section{Considerações finais}

Como resultados tivemos o retorno da satisfação imediata dos alunos com as atividades e o agradecimento de alguns, por vivenciarem uma dinâmica diferente daquelas do dia-a-dia. Os professores das escolas também se sentiram muito atraídos por todas as atividades, uma vez que também aprendem conceitos conosco, devido à carência que há sobre o assunto no ensino fundamental. Como uma forma de avaliar nossa atividade, foi pedido para que os que gostaram dessem um sinal de curtir, imitando a rede social Facebook, e tivemos 100\% de curtidas para as atividades.
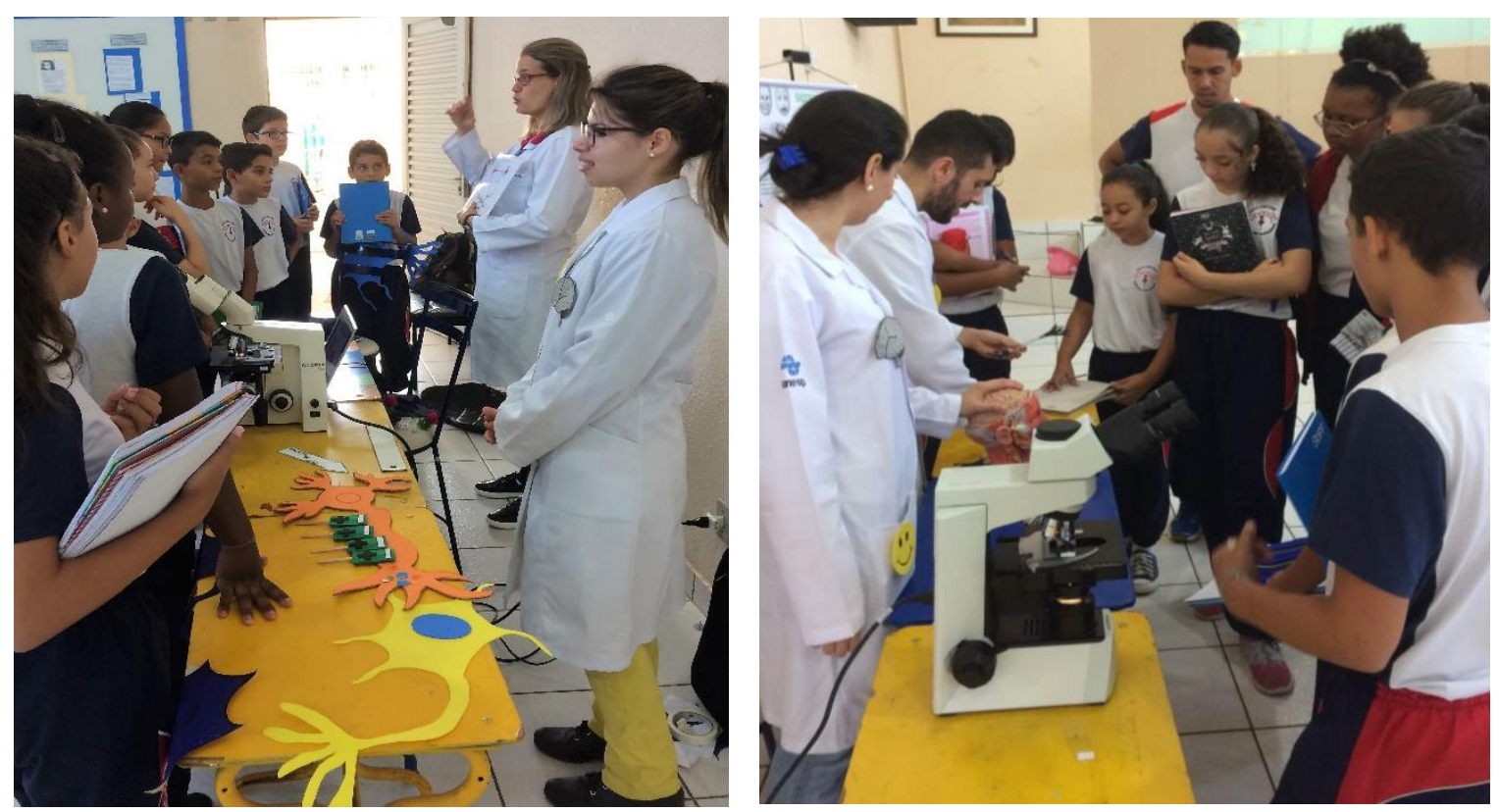

Figura 3. Detalhe das estações sobre o neurônio e seu funcionamento, e da estação sobre divisões do sistema nervoso. 
Cabe ressaltar que foi desafiador montar materiais e atividades que dialogassem com o público infantil. Os alunos de graduação são muito criativos em buscar alternativas para as explicações, fazendo pesquisas em sites internacionais que divulgam o tema e adaptando ideias para que as atividades fiquem bem atrativas e próximas ao cotidiano. Em adição, os alunos voluntários e colaboradores mostraram-se muito gratos em divulgar os conhecimentos, por se sentirem agentes transformadores da sociedade. Apesar de ser cansativa a repetição dos temas nas estações em rodízio durante nossas apresentações, os voluntários ficaram muito felizes ao perceberem que os alunos haviam guardado as informações das estações durante a gincana de perguntas e respostas. Todos os materiais produzidos para as atividades durante as semanas de divulgação foram disponibilizados para eventuais aulas práticas da disciplina de fisiologia. Em adição, os professores ensino do fundamental que se interessaram em reproduzir alguma das atividades receberam todas as orientações sobre os seus objetivos e como realizá-la. Os arquivos com as palavras cruzadas, caça-palavras, labirintos e figuras para colorir foram compartilhados com a coordenação das instituições visitadas para utilização por outros alunos ao longo das atividades da escola, não apenas durante nossa visita. As atividades mais simples de serem reproduzidas nas escolas foram copiadas e entregues para a coordenação dos locais que nos receberam. Em conclusão, as atividades de extensão foram muito satisfatórias e recompensadoras, tanto para nós que as desenvolvemos, como para a população que participou.

\section{Agradecimentos}

Agradecemos à Pró-Reitoria de Extensão Universitária da Universidade Estadual Paulista "Júlio de Mesquita Filho" (PROEX-UNESP) pelos recursos concedidos e o apoio da diretoria do Instituto de Biociências de Botucatu. Os autores também agradecem a receptividade da direção, professores, alunos, pais e amigos do Núcleo Assistencial Joanna de Ângelis, bem como a direção, funcionários e crianças do Projeto Preservando o Futuro de Rubião Junior da Ação da Cidadania do município de Botucatu, São Paulo, que participaram das ações.

\section{Contribuição de cada autor}

As autoras J. I. F. G, A. C. I. K. e S. M. N. colaboraram no planejamento das atividades para as exposições, desenvolveram algumas das atividades em campo, orientaram os alunos envolvidos. A autora J. I. F. G atuou na coordenação do projeto e escreveu a versão final, assim como a revisão intelectual crítica do artigo.

\section{Referências}

Abushouk, A. I., \& Duc, N. M. (2016). Curing neurophobia in medical schools: Evidence-based strategies. Medical Education Online, 21(1), 32476.

Buonanotte, M. C., Riveros, M., Villate, S., Beltramini, C., \& Buonanotte, C. F. (2016). Neurofobia o analfabetismo neurológico. Neurología Argentina, 8(1), 3-7.

Candotti, E. (2202). Ciência na educação popular. In L. Massarani, I. C. Moreira, \& F. Brito (Orgs.). Ciência e público: Caminhos da divulgação científica no Brasil. (pp. 15-24). Rio de Janeiro: Casa da Ciência.

Ekman, P., \& Friesen, W. V. (1971). Constants across cultures in the face and emotion. Journal of Personality and Social Psychology, 17(2), 124-129.

Filipin, G., Nunes, T. T., dos Santos Martins, A., Sosa, P. M., \& Mello-Carpes, P. B. (2014). Neuroblitz: Uma proposta de divulgação da neurociência na escola. Revista Ciência em Extensão, 10(3), 69-76.

Fragel-Madeira, L., \& Aranha, G. (2012). Divulgação e alfabetização científica: O papel do pesquisador na difusão do conhecimento científico. In, G. Aranha \& A. Sholl-Franco (Orgs.). Caminhos da Neuroeducação. (pp. 119-128). 2. ed. Rio de Janeiro: Ciências e Cognição. 
Herculano-Houzel, S. (2002). Do you know your brain? A survey on public neuroscience literacy at the closing of the decade of the brain. The Neuroscientist, 8(2), 98-110.

Lent, R. (2009). Brincar com a ciência em qualquer idade. Entrevista cedida a Marina Ramalho. Rio de Janeiro: FIOCRUZ. Recuperado de http://www.fiocruz.br/brasiliana/cgi/cgilua.exe/sys/start.htm?infoid=545\&sid=31

Lindell, A. K., \& Kidd, E. (2013). Consumers favor "right brain" training: The dangerous lure of neuromarketing. Mind, Brain, and Education, 7(1), 35-39.

Pasquinelli, E. (2012). Neuromyths: Why do they exist and persist? Mind, Brain, and Education, 6(2), 89-96.

Racine, E., Waldman, S., Rosenberg, J., \& Illes, J. (2010). Contemporary neuroscience in the media. Social science \& medicine, 71(4), 725-733.

Rolim, A. A. M., Guerra, S. S. F., \& Tassigny, M. M. (2008). Uma leitura de Vygotsky sobre o brincar na aprendizagem e no desenvolvimento infantil. Revista Humanidades, 23(2), 176-180.

Santos-Lobato, B. L., Magalhães, Á. B., Moreira, D. G., Farias, F. P., Porto, L. K., Pereira, R. B., ... \& Braga, T. K. K. (2018). Neurophobia in Brazil: Detecting and Preventing a Global Issue. Revista Brasileira de Educação Médica, 42(1), $121-128$.

Sholl-Franco, A., Miguel, A. C., \& Guedes, S. (2015). Desenhando emoções. Rio de Janeiro: Ciência e Cognição.

Zardetto-Smith, A. M., Mu, K., Phelps, C. L., Houtz, L. E., \& Royeen, C. B. (2002). Brains rule! Fun=learning=neuroscience literacy. The Neuroscientist, 8(5), 396-404.

Como citar este artigo:

De Gobbi, J. I. F., Kiss, A. C. I., \& Nishida, S. M. (2020). Semana de conscientização sobre o cérebro em Botucatu: Interação universidade e ensino fundamental. Revista Brasileira de Extensão Universitária, 11(1), 87-95. https://periodicos.uffs.edu.br/index.php/RBEU/article/view/10677/pdf
} 